\title{
Dynamic finite-size scaling after a quench at quantum transitions
}

\author{
Andrea Pelissetto, ${ }^{1,}{ }^{*}$ Davide Rossini, ${ }^{2}$ and Ettore Vicari ${ }^{2}$ \\ ${ }^{1}$ Dipartimento di Fisica dell'Università di Roma "La Sapienza" and INFN, Sezione di Roma I, I-00185 Roma, Italy \\ ${ }^{2}$ Dipartimento di Fisica dell'Università di Pisa and INFN, Largo Pontecorvo 3, I-56127 Pisa, Italy
}

(Received 13 April 2018; published 31 May 2018)

\begin{abstract}
We present a general dynamic finite-size scaling theory for the quantum dynamics after an abrupt quench, at both continuous and first-order quantum transitions. For continuous transitions, the scaling laws are naturally ruled by the critical exponents and the renormalization-group dimension of the perturbation at the transition. In the case of first-order transitions, it is possible to recover a universal scaling behavior, which is controlled by the size behavior of the energy gap between the lowest-energy levels. We discuss these findings in the framework of the paradigmatic quantum Ising ring, and support the dynamic scaling laws by numerical evidence.
\end{abstract}

DOI: 10.1103/PhysRevE.97.052148

\section{INTRODUCTION}

Understanding the quantum evolution of many-body systems is an outstanding and intensely debated problem, starting from the dawn of quantum mechanics. Up to the beginning of the new millennium, issues related to this topic were mostly considered as merely academic [1-6]. However, the recent technological breakthroughs in the realization, control, and readout of the coherent dynamics of isolated quantum manybody systems for a significant amount of time (as for ultracold atoms or trapped ions) have changed this point of view $[7,8]$. As a matter of fact, they have catalyzed a huge body of scientists, working both on the theoretical and the experimental side. In this context, the so-called quantum quench probably represents the simplest protocol in which a system can be naturally put in out-of-equilibrium conditions [9-14]

Several interesting issues have been deeply scrutinized in the recent years for the quantum dynamics after a quench, stimulating a fervid scientific activity. They include the long-time relaxation and the consequent spreading of correlations, the statistics of the work, the mutual interplay of interactions and disorder, aging and coarsening properties, short-time dynamic scaling, and dynamical phase transitions, to mention some of the most representative ones (see, e.g., Refs. [15-19] and references therein). In this paper we focus on a further issue related to quantum quench dynamics, that is the emergence of a dynamic finite-size scaling (DFSS) in the quantum dynamics of an isolated many-body system after a quench in proximity of a quantum phase transition. We put forward a DFSS theory in the appropriate limit, which is valid at generic continuous quantum transitions (CQTs) and at first-order quantum transitions (FOQTs).

Before giving the details of our investigation, we formally introduce the setting. A quench protocol is generally performed within a family of Hamiltonians, that are written as the sum of two noncommuting terms:

$$
H(\lambda)=H_{u}+\lambda P .
$$

\footnotetext{
*Authors are provided in alphabetical order.
}

The tunable parameter $\lambda$ enables us to modify the strength of the perturbation $P$, e.g., a magnetic field term in a system of interacting spins, with respect to the unperturbed Hamiltonian $H_{u}$. The idea of a quantum quench is to prepare the system in the ground state of Hamiltonian (1) associated with an initial value $\lambda_{0}$, that is $|\Psi(0)\rangle \equiv\left|0_{\lambda_{0}}\right\rangle$, and then suddenly change the parameter to $\lambda \neq \lambda_{0}$. The resulting dynamic problem corresponds to that of the quantum evolution driven by the Hamiltonian $H(\lambda)$, starting from the particular initial condition of the ground state of $H\left(\lambda_{0}\right)$, that is $|\Psi(t)\rangle=e^{-i H(\lambda) t}\left|0_{\lambda_{0}}\right\rangle$ (hereafter we will adopt units of $\hbar=k_{B}=1$ ).

We are interested in the quench dynamics occurring within the critical regime of a quantum transition. Thus, $H_{u}$ describes a system at a CQT or a FOQT. In the following we discuss the interplay between the quench parameters $\lambda_{0}$ and $\lambda$ and the finite size $L$ of the system, assuming that both the initial $\left(\lambda_{0}\right)$ and final $(\lambda)$ parameters keep the system close to the quantum transition point. For this purpose, we define a DFSS limit as the large-size and large-time limit, keeping the appropriate scaling variables fixed. At CQTs such scaling variables are the combinations $t L^{-z}, \lambda_{0} L^{y_{\lambda}}$, and $\lambda L^{y_{\lambda}}$, where $z$ and $y_{\lambda}$ are suitable critical exponents. Namely, $z$ is the dynamic exponent associated with the critical behavior of the low-energy spectrum, and $y_{\lambda}$ is the renormalization-group (RG) dimension of the parameter $\lambda$. At FOQTs power laws may turn into exponential laws related to the size dependence of the energy gap.

The DFSS that we put forward is validated within the quantum Ising chain, the paradigmatic model undergoing FOQTs and CQTs, when varying its parameters. In particular, we consider quench protocols associated with variations of the longitudinal magnetic field coupled to the order-parameter spin operators. We present analytical and numerical results for the off-equilibrium behavior of several quantities, including the magnetization; the Loschmidt echo, which measures the overlap between the evolved state and the initial state of the system; and the bipartite entanglement entropy, quantifying the quantum correlations between different spatial parts of the system.

The paper is organized as follows. In Sec. II we put forward the general DFSS theory for the quantum evolution after a quench at CQTs. In Sec. III we specialize the discussion to the 
quantum Ising ring: we introduce the model and we report the expected scaling behavior of the magnetization and two-point correlations. We also thoroughly discuss the scaling of the Loschmidt echo and of the bipartite entanglement entropy. The predicted asymptotic behaviors are then verified numerically. In Sec. IV we analytically derive the scaling functions for the Ising ring along the FOQT line, by employing a two-level truncation of the system's Hilbert space, and numerically show that they are asymptotically exact, up to exponential corrections in the system size. Finally, Sec. V presents a summary, our conclusions, and future perspectives.

\section{DYNAMIC SCALING THEORY AT A CQT}

We first recall that the theory of finite-size scaling (FSS) at quantum transitions is well established; see, e.g., Ref. [20] and references therein. Briefly speaking, one can assume that a $d$-dimensional quantum transition [21] is characterized by two relevant parameters $\mu$ and $\lambda$, such that they vanish at the critical point, with RG dimension $y_{\mu}$ and $y_{\lambda}$, respectively. The asymptotic FSS behavior of a generic observable $O$ with RG dimension $y_{o}$ is thus given by

$$
O(L, \mu, \lambda) \approx L^{-y_{o}} \mathcal{O}\left(\mu L^{y_{\mu}}, \lambda L^{y_{\lambda}}\right),
$$

where $L$ denotes the linear size of the $d$-dimensional system under investigation.

In order to characterize the dynamic behavior after a quench, we extend the FSS framework to the quench case. We consider a Hamiltonian

$$
H(\lambda)=H_{c}+\lambda P,
$$

where $H_{c}$ is critical (for $\lambda=0$ the system undergoes a CQT) and $\lambda$ is a control parameter associated with the relevant perturbation $P$. In the quench protocol we start from the ground state of $H\left(\lambda_{0}\right)$. Then, at the reference time $t=t_{0}=0$, we suddenly switch the coupling from $\lambda_{0}$ to $\lambda$ and follow the subsequent evolution of the system. In the following, we always assume that $\lambda$ and $\lambda_{0}$ are sufficiently small, so that the system is always close to the quantum transition point.

In order to write down the dynamic scaling ansatz for the postquench behavior of the system, we introduce the scaling variables

$$
\kappa(\lambda)=\lambda L^{y_{\lambda}}, \quad \theta=t L^{-z}
$$

where $t$ is the time, and $z$ is the dynamic exponent characterizing the behavior of the energy differences of the lowestenergy states and, in particular, the gap $\Delta \sim L^{-z}$. A DFSS should emerge in the infinite-volume limit $L \rightarrow \infty$, keeping $\theta, \kappa_{0} \equiv \kappa\left(\lambda_{0}\right)$, and $\kappa \equiv \kappa(\lambda)$ fixed. Then, a generic global observable $O$, the RG dimension of which at the critical point is $y_{o}$, is expected to behave as

$$
O\left(t, L, \lambda_{0}, \lambda\right) \approx L^{-y_{o}} \mathcal{O}\left(\theta, \kappa_{0}, \kappa\right)=L^{-y_{o}} \mathcal{O}\left(\theta, \kappa, \delta_{\lambda}\right),
$$

where

$$
\delta_{\lambda} \equiv \frac{\kappa}{\kappa_{0}}-1=\frac{\lambda}{\lambda_{0}}-1 .
$$

An analogous scaling is expected for the correlation functions. The corrections to the above asymptotic DFSS laws are expected to decay as negative powers of the size $L$. In the
RG language, they may, for example, arise from the presence of irrelevant perturbations at the fixed point controlling the critical behavior. Note that the equilibrium (ground-state) FSS behavior must be recovered in the limit $\delta_{\lambda} \rightarrow 0$. We also mention that a similar dynamic scaling behavior was also proposed, and verified, in the context of trapped bosonic gases (confined by a harmonic potential) for quench protocols associated with the size of the trap [22].

The Loschmidt amplitude quantifies the deviation of the postquench state at time $t>0$ from the initial state before the quench. It is defined as the overlap

$$
A(t)=\left\langle 0_{\lambda_{0}} \mid \Psi(t)\right\rangle=\left\langle 0_{\lambda_{0}}\left|e^{-i H(\lambda) t}\right| 0_{\lambda_{0}}\right\rangle .
$$

We introduce the rate function

$$
Q(t)=-\ln |A(t)|^{2},
$$

which provides information on the so-called Loschmidt echo [in the following we refer to $Q(t)$ as the Loschmidt echo]. Note that $Q(t)=0$ implies the restoration of the initial quantum state. We conjecture that the time dependence of $Q(t)$ after the quench obeys the DFSS behavior:

$$
Q\left(t, L, \lambda_{0}, \lambda\right) \approx \mathcal{Q}\left(\theta, \kappa, \delta_{\lambda}\right) .
$$

We may also evaluate the average work [23],

$$
\mathcal{L} \equiv\langle W\rangle=E-E_{0},
$$

which is necessary to perform the instantaneous quench at $t=0$. The energy $E$ injected into the system by the quench is given by the expectation value of the postquench Hamiltonian $H(\lambda)$ on the initial (prequench) state $\left|0_{\lambda_{0}}\right\rangle$ :

$$
\begin{aligned}
E & =\left\langle 0_{\lambda_{0}}|H(\lambda)| 0_{\lambda_{0}}\right\rangle \\
& =\left\langle 0_{\lambda_{0}}\left|H\left(\lambda_{0}\right)\right| 0_{\lambda_{0}}\right\rangle+\left(\lambda-\lambda_{0}\right)\left\langle 0_{\lambda_{0}}|P| 0_{\lambda_{0}}\right\rangle .
\end{aligned}
$$

Since the initial energy is $E_{0}=\left\langle 0_{\lambda_{0}}\left|H\left(\lambda_{0}\right)\right| 0_{\lambda_{0}}\right\rangle$, we obtain

$$
\mathcal{L}=E-E_{0}=\left(\lambda-\lambda_{0}\right)\left\langle 0_{\lambda_{0}}|P| 0_{\lambda_{0}}\right\rangle .
$$

In the DFSS limit, we can exploit the equilibrium FSS behavior, Eq. (2), to evaluate the matrix element $\left\langle 0_{\lambda_{0}}|P| 0_{\lambda_{0}}\right\rangle$. Assuming that $P=\sum_{x} P_{x}$ is a sum of local terms, we have

$$
\mathcal{L} \approx\left(\lambda-\lambda_{0}\right) L^{d-y_{p}} f_{p}\left(\kappa_{0}\right),
$$

where $f_{p}$ is the equilibrium FSS function associated with the observable $P / L^{d}$. Taking into account the relation [21]

$$
y_{p}+y_{\lambda}=d+z
$$

between the RG dimensions of $\lambda$ and of the associated perturbation $P$, the scaling behavior of the work $\mathcal{L}$ can be eventually written as

$$
\mathcal{L} \approx L^{-z} \delta_{\lambda} \kappa_{0} f_{p}\left(\kappa_{0}\right) .
$$

We may also consider the large-volume limit of the above scaling behaviors. If $O$ is an intensive variable that has a finite limit for $L \rightarrow \infty$ at $\lambda, \lambda_{0} \neq 0$, from Eq. (5) we obtain the infinite-volume dynamic scaling behavior,

$$
O\left(t, L \rightarrow \infty, \lambda_{0}, \lambda\right) \approx \lambda^{-y_{o} / y_{\lambda}} \mathcal{O}_{\infty}\left(\lambda^{z / y_{\lambda}} t, \delta_{\lambda}\right),
$$

which is valid for $\lambda, \lambda_{0} \rightarrow 0$, and $t \rightarrow \infty$, keeping $\lambda^{z / y_{\lambda}} t$ and $\delta_{\lambda}$ fixed. For $L \rightarrow \infty$, the work grows as the volume, which 
implies

$$
\begin{aligned}
f_{p}\left(\kappa_{0}\right) & \sim \kappa_{0}^{y_{p} / y_{\lambda}}, \quad \kappa_{0} \rightarrow \infty, \\
\mathcal{L} & \sim L^{d} \delta_{\lambda} \lambda_{0}^{1+y_{p} / y_{\lambda}} .
\end{aligned}
$$

We finally remark that the above DFSS arguments can be straightforwardly extended to more complicated quench protocols, for example when they involve changes of both relevant parameters $\mu$ and $\lambda$.

\section{SCALING ACROSS THE CQT OF THE ISING RING}

To fix the ideas, we now demonstrate how a DFSS behavior emerges along a sudden quench of the simplest paradigmatic quantum many-body system, exhibiting a nontrivial zerotemperature behavior: the one-dimensional quantum Ising chain in the presence of a transverse field. Namely, we show how to describe the interplay between the various parameters of the quench protocol and the finite size of the system in an appropriate DFSS limit.

\section{A. Hamiltonian model and quench protocol}

The Hamiltonian of a quantum Ising ring is

$$
H_{\mathrm{Is}}=-\sum_{x=1}^{L}\left[J \sigma_{x}^{(3)} \sigma_{x+1}^{(3)}+g \sigma_{x}^{(1)}\right],
$$

where, on each site $x$ of the chain, the spin variables $\sigma \equiv\left(\sigma^{(1)}, \sigma^{(2)}, \sigma^{(3)}\right)$ are the Pauli matrices, and $\sigma_{L+1}=\sigma_{1}$ denotes periodic boundary conditions. The parameters $J$ and $g$, respectively, denote a ferromagnetic nearest-neighbor interaction (we assume $J=1$ ) and the transverse field strength (we assume $g>0$ ). A CQT occurs at $g=1$, separating a disordered $(g>1)$ from an ordered $(g<1)$ quantum phase [21]. This CQT belongs to the two-dimensional Ising universality class with critical exponents $v=1, \eta=1 / 4$, and $z=1$, which are associated with the diverging length scale, the behavior of the two-point function at the critical point, and the energy gap at the transition, respectively.

In the following, we wish to analyze the quantum dynamics arising from a quench protocol associated with an external magnetic field along the longitudinal direction. We thus add a magnetic perturbation $P=-\sum_{x} \sigma_{x}^{(3)}$ to Eq. (18), that is, we consider

$$
H(\lambda)=H_{\mathrm{Is}}-\lambda \sum_{x=1}^{L} \sigma_{x}^{(3)} .
$$

In the quench protocol we start at $t=0$ from the ground state of the system, at the parameter value $\lambda_{0}$, and suddenly change it to $\lambda \neq \lambda_{0}$. Then we consider the time evolved state $|\Psi(t)\rangle$. The quantum evolution is characterized by the time dependence of observables computed at $t>0$, such as the magnetization $M$ and the connected correlation function

$$
\begin{array}{r}
M\left(t, L, \lambda_{0}, \lambda\right)=\frac{1}{L}\left\langle\Psi(t)\left|\sum_{x=1}^{L} \sigma_{x}^{(3)}\right| \Psi(t)\right\rangle, \\
G_{c}\left(x-y, t, L, \lambda_{0}, \lambda\right)=\left\langle\Psi(t)\left|\sigma_{x}^{(3)} \sigma_{y}^{(3)}\right| \Psi(t)\right\rangle_{c},
\end{array}
$$

respectively. We used the translation invariance to infer the space dependence of $G_{c}$.

\section{B. Scaling behavior}

We now focus on the dynamics arising from the quench protocol when the unperturbed Hamiltonian $H_{\mathrm{Is}}$ is at the CQT point, that is, for $g=1$. The parameters $\lambda_{0}$ and $\lambda$ are assumed to be sufficiently small to maintain the system in the critical region.

In the DFSS theory put forward in Sec. II, the relevant scaling variables are reported in Eq. (4). Specializing to the one-dimensional Ising model, we have

$$
y_{\lambda}=(d+z+2-\eta) / 2=15 / 8, \quad z=1 .
$$

The magnetization (20) obeys the scaling behavior

$$
M\left(t, L, \lambda_{0}, \lambda\right) \approx L^{-\beta / \nu} \mathcal{M}\left(\theta, \kappa, \delta_{\lambda}\right),
$$

where $\beta$ denotes the magnetization critical exponent, and thus $\beta / \nu=1 / 8$. One might also consider other observables, such as the connected two-point function (21), that should scale as

$$
G_{c}\left(x, t, L, \lambda_{0}, \lambda\right) \approx L^{-\eta / v} \mathcal{G}\left(X, \theta, \kappa, \delta_{\lambda}\right),
$$

where $\eta / v=1 / 4$ and $X \equiv x / L$. The corresponding length scale, defined, for example, from the second moment of $G_{c}$, is expected to behave as

$$
\xi\left(t, L, \lambda_{0}, \lambda\right) \approx L \Xi\left(\theta, \kappa, \delta_{\lambda}\right) .
$$

The work associated with the quench is expected to scale as in Eq. (15). We note that, for a sudden change of the sign of the magnetic field, i.e., for $\lambda=-\lambda_{0}$ (correspondingly, $\delta_{\lambda}=-2$ ), the ground-state energies of the initial and final Hamiltonians are equal, due to the symmetry of the Ising ring. Thus, in this case the work $\mathcal{L}$ also provides the energy difference between the state of the system and that of the ground state during the postquench quantum evolution.

The asymptotic scaling behaviors are expected to be approached with power-law suppressed corrections. In the quantum Ising ring without boundaries (i.e., with periodic boundary conditions) scaling corrections to the equilibrium FSS laws [20] usually decay as $L^{-\omega}$, where $\omega=2$ is the leading scaling-correction exponent [24]. As we shall observe in Sec. IIIC, corrections are compatible with an $L^{-2}$ behavior also in out-of-equilibrium conditions $(\theta \neq 0)$. However, we cannot exclude the appearance in the DFSS case of new types of scaling corrections that decay with a smaller power of the lattice size, originating, for instance, from the breaking of the time-translation invariance due to the initial condition of the quench protocol.

Analogous DFSS laws can be written for quenches arising from the sudden change of a local perturbation. For instance, one can replace Eq. (19) with

$$
H(\lambda)=H_{\mathrm{Is}}-\lambda \sigma_{1}^{(3)},
$$

where the perturbation is on a single site only. In this case, the local magnetization on site $x$ should behave as

$$
M_{x}\left(t, L, \lambda_{0}, \lambda\right) \approx L^{-\beta / \nu} \mathcal{M}_{l}\left(x_{p} / L, \theta, \kappa, \delta_{\lambda}\right),
$$

where the RG dimension entering the definition of $\kappa$ [see Eq. (4)] is $y_{\lambda}=1 / 2$ (see Ref. [25] and references therein). 

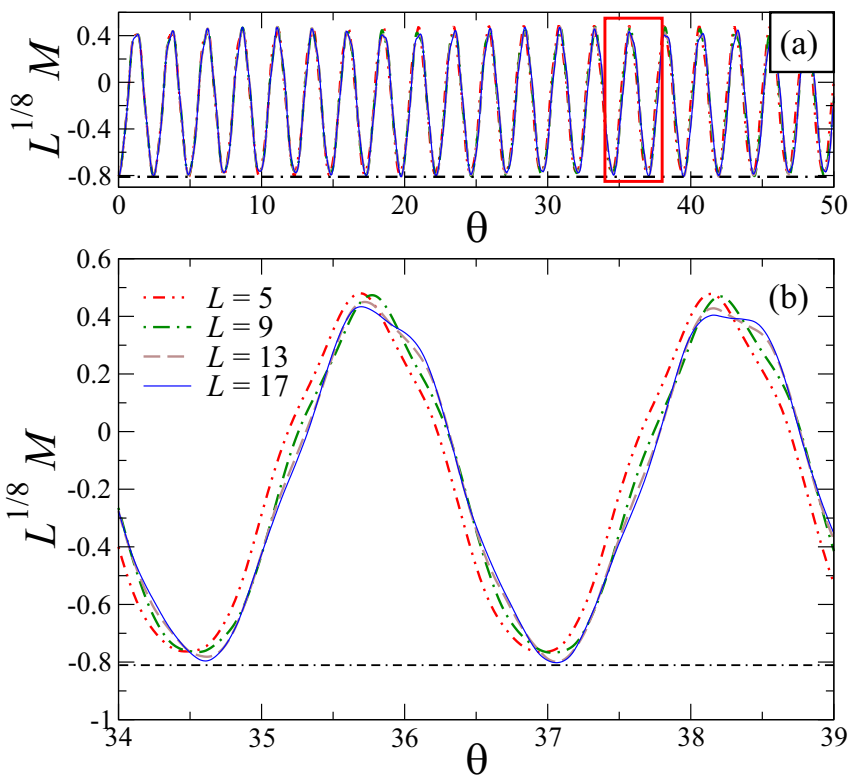

FIG. 1. Rescaled magnetization for the quantum critical Ising ring vs the rescaled time $\theta$, for $\kappa=1, \delta_{\lambda}=-2$, and several values of $L$. Panel (b) shows a magnification of panel (a), for $34 \leqslant \theta \leqslant 39$ (red box). The horizontal dot-dashed line indicates the infinite-size limit value of the static magnetization at $\kappa_{0}$. Notice a clear trend towards an asymptotic oscillatory function with increasing $L$, thus confirming DFSS.

As a consequence, the DFSS behavior of its spatial average should be

$$
M\left(t, L, \lambda_{0}, \lambda\right) \approx L^{-\beta / v} \mathcal{M}_{a}\left(\theta, \kappa, \delta_{\lambda}\right)
$$

Finally, we point out that the above DFSS arguments apply also to the case in which the quench protocol is associated with a transverse magnetic field, i.e., with the perturbation $P_{t}=\sum_{x=1}^{L} \sigma_{x}^{(1)}$. In this case, the RG dimension of the perturbation is simply $y_{\lambda}=1 / v=1$. For a transverse field the magnetization vanishes by symmetry, but one may consider the two-point function and the corresponding correlation length. We mention that some results for quantum quenches involving the transverse field in the infinite-size limit are reported in Refs. [26-28]. More general quantum quenches in the infinitevolume limit are also discussed in Ref. [29].

\section{Numerical results}

In order to validate the DFSS predictions outlined above, we now present the results of numerical simulations of the dynamics of the Ising ring with Hamiltonian (19), after a sudden quench in $\lambda$. For our purposes, it has been sufficient to consider systems of moderate sizes (up to $L \approx 23$ sites). An exact diagonalization approach has been used for systems with $L \leqslant 13$, while Lanczos diagonalization followed by a fourthorder Suzuki-Trotter decomposition of the unitary-evolution operator, with time step $d t=10^{-2}$, was employed for larger sizes $(14 \leqslant L \leqslant 23)$.

We start from the analysis of the magnetization defined in Eq. (20). The numerical data of Fig. 1, corresponding to fixed values of $\kappa=1$ and $\delta_{\lambda}=-2$, show that the product
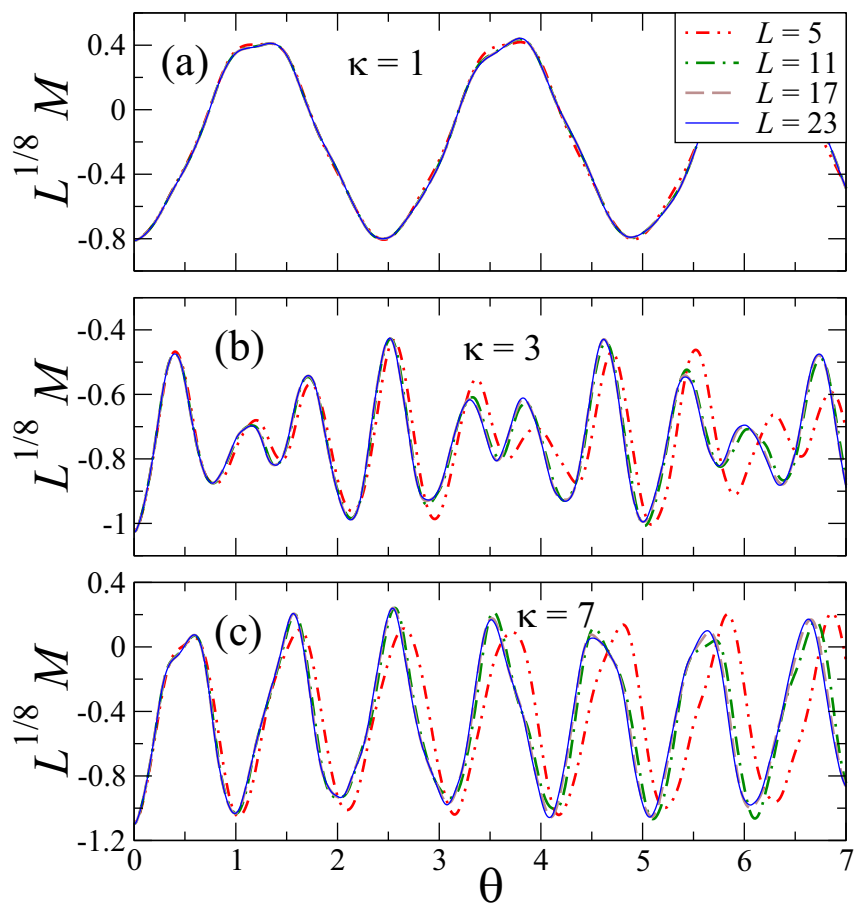

FIG. 2. Same plot as in Fig. 1, but for three different values of $\kappa: 1$ (a), 3 (b), and 7 (c). In all cases the convergence to a scaling behavior, in the large- $L$ limit, is clearly visible. Data in panel (a) are for the same parameters as in Fig. 1, but on a different time scale.

$L^{1 / 8} M$, as a function of the rescaled time $\theta$, clearly approaches an asymptotic function with increasing $L$. This confirms the DFSS prediction (23). Convergence seems to be notably fast with $L$. An oscillatory behavior, with the emergence of wiggles in proximity of the peaks, clearly appears already for a moderately large size. However, when zooming in the figure, a rather complicated pattern emerges, signaling that the dynamics cannot be trivially obtained by using an effective few-level description of the system. As we shall see later, it is, however, possible to extrapolate an asymptotic scaling behavior at any value of $\theta$, which takes into account all these features.

Notice also that the pseudosinusoidal trend persists at long times, without appreciable damping in the oscillation amplitude. Indeed, we checked that the magnetization comes back periodically in time to a value that is very close (although not equal) to the initial value, the extrapolated infinite-size limit of which is plotted as an horizontal line in the figure. The absence of a stationary large- $\theta$ limit reflects the lack of thermalization, which is expected for this kind of quench in the longitudinal field of the otherwise integrable Ising ring.

In Fig. 2 we change the value of $\kappa$, keeping $\delta_{\lambda}=-2$. Similar patterns emerge, all of them exhibiting convergence to an asymptotic function, thus agreeing with the DFSS prediction (23). It is tempting to compare the emerging temporal features with those observed in Fig. 1: for example, at $\kappa=3$, we observe a less regular pattern, with a (pseudo-) periodicity which differs from the previous case. For $\kappa=7$ a more regular trend seems to reappear, although with a much smaller period. This behavior with $\kappa$ has to be ascribed to the degree of commensurability of the injected energy with the spectrum of the system. Notice 


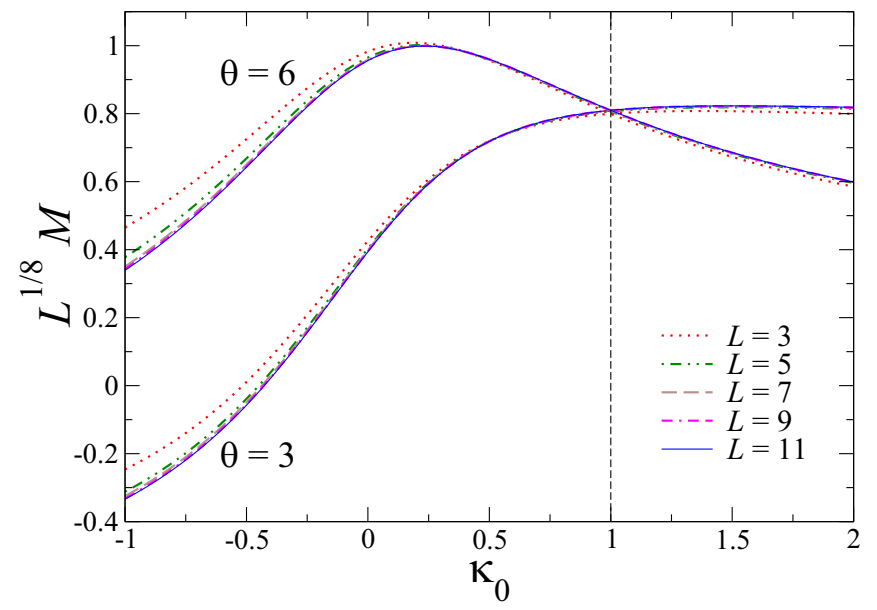

FIG. 3. Magnetization for fixed $\kappa=1$ and for two different rescaled times $\theta$. The curves are plotted against the rescaled parameter $\kappa_{0}$, which is used to compute the initial state. Notice that, at $\kappa_{0}=\kappa=1$, the equilibrium behavior is recovered (vertical dashed line). As before, we observe that the curves at different size approach an asymptotic function, in accordance with our DFSS theory.

also that, for fixed $\theta$, the approach to the asymptotic scaling behavior appears to be slower for larger $\kappa$.

DFSS can also be checked as a function of the initial state, that is, of the value of $\kappa_{0}$ before the quench. This is what we have done in Fig. 3, where we display the magnetization after a quench at fixed rescaled time $\theta$ and $\kappa=1$, as a function of $\kappa_{0}$ (thus, $\delta_{\lambda}=\kappa / \kappa_{0}-1=\lambda / \lambda_{0}-1$ is now changing). Two values of $\theta$ are shown. The various curves spotlight the emergence of a scaling behavior, in a way similar to the previous cases as a function of $\theta$. Obviously they intersect at the equilibrium point, which is located at $\kappa_{0}=\kappa$, i.e., $\delta_{\lambda}=0$.

We have performed additional numerical simulations (not shown) for several other choices of the scaling variables $\kappa_{0}, \delta_{\lambda}$, and $\theta$, confirming a similar fast convergence with $L$ to the asymptotic functions, obeying the DFSS theory.

Let us now switch to the analysis of the Loschmidt echo $Q(t)$ defined in Eq. (8). Numerical data are plotted in Fig. 4; the emerging pattern is similar to that of the magnetization, although quantitatively presenting different features. The data fully support the DFSS predicted by the scaling equation (9). We note in particular the evidence of quasicomplete revivals of the quantum states along the quantum evolution, when $Q(t) \ll 1$.

To better check the convergence to the asymptotic scaling behavior in the $L \rightarrow \infty$ limit, we have explicitly analyzed the dependence of the various quantities with the size, keeping the scaling variables fixed. The corresponding data for the magnetization and the Loschmidt echo, plotted as functions of $1 / L^{2}$, are displayed in Fig. 5, where we highlight a few representative values of $\kappa$ and $\theta$. Finite-size corrections appear to be substantially consistent with an $L^{-2}$ behavior, which is the trend expected for the homogeneous Ising ring at equilibrium [24] (as a matter of fact, we explicitly checked the excellent quality of an $L^{-2}$ fit at $\theta=0$ ). Notice that, on the scale of the figure, the dependence on $L$ is barely visible, except for $\kappa=7$, where finite-size corrections are more evident.

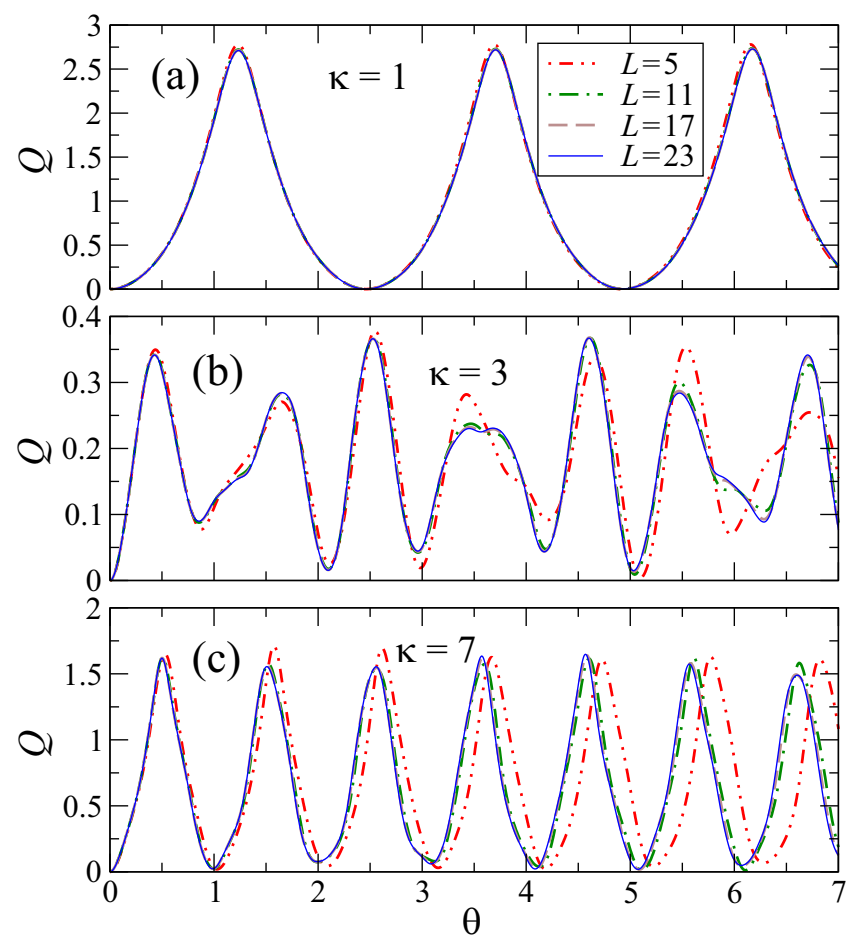

FIG. 4. Temporal behavior of the Loschmidt echo $Q(t)$ defined in Eq. (8) for $\delta_{\lambda}=-2$, and three different values of $\kappa: 1$ (a), 3 (b), and 7 (c). Convergence to a scaling function, in the large- $L$ limit, is clearly visible.

Finally, we consider the time evolution of the entanglement entropy of bipartitions of the system, which quantifies the amount of quantum correlations that are present between the two parts of the chain. These are operatively calculated by means of the following procedure: we divide the chain into two connected parts of length $\ell_{A}$ and $L-\ell_{A}$ (for the sake of clarity, we always take $\ell_{A}=L / 2$ ), and compute the so-called
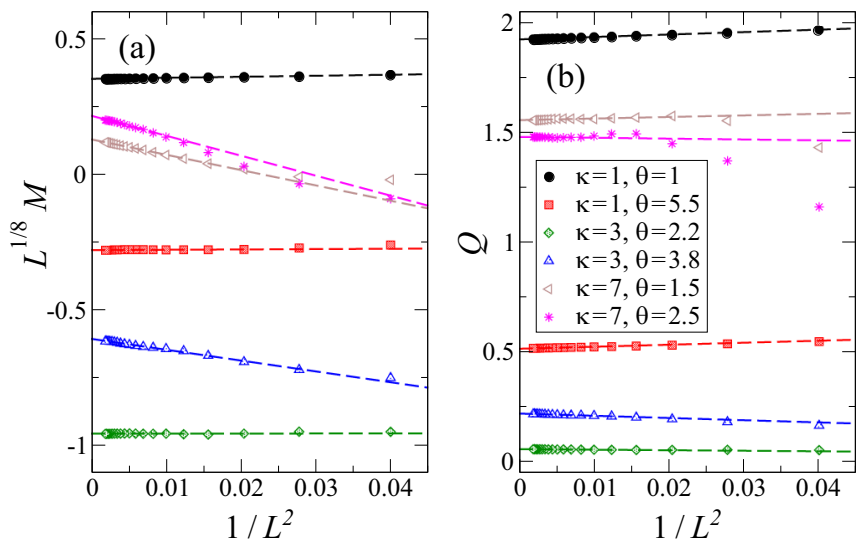

FIG. 5. Behavior of the rescaled magnetization $L^{1 / 8} M($ a) and of the Loschmidt echo $Q(t)$ (b) with the system size, for different values of $\kappa$ and $\theta$, as indicated in the legend (data of Figs. 2 and 4 have been used). Data are plotted against $1 / L^{2}$ : straight dashed lines denote $O\left(L^{-2}\right)$ fits of the numerical values (symbols) for large $L$, and are plotted to guide the eye towards the extrapolated infinite-size limit. 


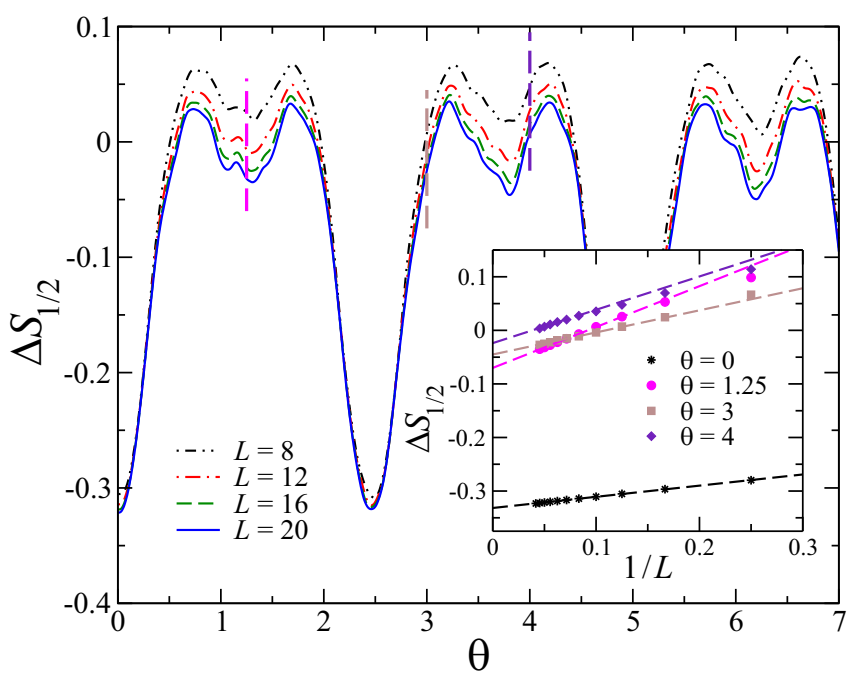

FIG. 6. Temporal behavior of the entanglement entropy for a balanced bipartition of $L / 2$ sites, after a quench of the longitudinal field in the quantum Ising ring. The inset displays the convergence with $L$ of the various curves (up to $L=22$ ), for three values of $\theta \neq 0$ (see the long-dashed lines in the main panel), plotted against $1 / L$. Additionally, black stars denote data corresponding to the equilibrium condition $\theta=0$ (up to $L=24$ ). Dashed lines are numerical fits of the data at the largest available $L$.

von Neumann (vN) entropy:

$$
S\left(\ell_{A}, L\right)=S\left(L-\ell_{A}, L\right)=-\operatorname{Tr}\left[\rho_{A} \ln \rho_{A}\right] .
$$

Here $\operatorname{Tr}[\cdot]$ denotes the trace operation, while $\rho_{A}=\operatorname{Tr}_{L \backslash A}[|\psi\rangle\langle\psi|]$ is the reduced density matrix of subsystem $A$, with $|\psi\rangle$ being the quantum state of the global chain. The asymptotic large- $L$ behavior of the ground-state bipartite entanglement entropy of the quantum Ising ring at the critical point $g=1$ and $\lambda=0$ is known to be [30-32]

$$
S_{c}\left(\ell_{A}, L\right)=\frac{1}{6}\left[\ln L+\ln \sin \left(\pi \ell_{A} / L\right)+e\right]+O\left(L^{-2}\right),
$$

where $e$ is a known constant. Definition (29) applies also to the time-dependent case, allowing us to compute the bipartite $\mathrm{vN}$ entropy $S\left(\ell_{A}, L, t, \lambda_{0}, \lambda\right)$ on the state $|\Psi(t)\rangle$ resulting after the quench at $t=0$. We consider, in particular, the case of a balanced bipartition, i.e., $\ell_{A} / L=1 / 2$. Extending equilibrium scaling arguments (see, e.g., Ref. [20]), we conjecture the DFSS behavior:

$$
\begin{aligned}
\Delta S_{1 / 2} & \equiv S\left(L / 2, L, t, \lambda_{0}, \lambda\right)-S_{c}(L / 2, L) \\
& \approx \mathcal{S}\left(\theta, \kappa, \delta_{\lambda}\right) .
\end{aligned}
$$

DFSS is nicely supported by the time dependence of the half-chain vN entropy, as shown in Fig. 6. We have also studied the rate of approach to the asymptotic regime. As spotlighted in the inset of Fig. 6, corrections to the asymptotic DFSS behavior (31) generally scale as $1 / L$. This is true both for the ground state of the initial Hamiltonian (see the black stars in the inset, corresponding to $\theta=0$ ) and for the evolved state (we report results for three different values of $\theta \neq 0$ ). These corrections, and in particular those at $\theta=0$ corresponding to the initial equilibrium ground state, are related to the so-called conical corrections [33]. They are expected to be generally
$O(1 / L)$ for the bipartite vN entanglement entropy around the CQT of the quantum Ising chain (see, e.g., Ref. [20] for a detailed discussion). Note, however, that finite-size corrections for the ground-state $\mathrm{vN}$ entropy decay as $1 / L^{2}$ at the CQT point $(g=1$ and $\lambda=0)$ for periodic boundary conditions [see Eq. (30)], where the leading conical correction cancels out. Our numerical data show that this cancellation does not occur for $\lambda \neq 0$.

\section{DYNAMIC FINITE-SIZE SCALING ALONG THE FOQT LINE}

In this section we extend the DFSS theory to FOQTs. Although the presentation refers to the Ising ring with Hamiltonians (19) or (26), the results apply quite straightforwardly to generic transitions.

For any $g<1$ (we assume $g>0$ ) the ground state of the Ising Hamiltonian (18) is doubly degenerate. The degeneracy is lifted by the introduction of a longitudinal field, such as that appearing in Eqs. (19) and (26). Therefore, $\lambda=0$ is a FOQT point, where the longitudinal magnetization $M=L^{-1} \sum_{x=1}^{L} M_{x}$, with $M_{x} \equiv\left\langle\sigma_{x}^{(3)}\right\rangle$, becomes discontinuous in the infinite-volume limit. The FOQT separates two different phases characterized by opposite values of the (spontaneous) magnetization $m_{0}$ given by [34]

$$
\lim _{\lambda \rightarrow 0^{ \pm}} \lim _{L \rightarrow \infty} M= \pm m_{0}, \quad m_{0}=\left(1-g^{2}\right)^{1 / 8} .
$$

In a finite system of size $L$, the two lowest states are superpositions of two magnetized states $|+\rangle$ and $|-\rangle$ such that

$$
\left\langle \pm\left|\sigma_{x}^{(3)}\right| \pm\right\rangle= \pm m_{0}
$$

for all $x$. Due to tunneling effects, the energy gap $\Delta$ vanishes exponentially as $L$ increases [34,35]:

$$
\Delta(L) \approx 2\left(\frac{1-g^{2}}{\pi L}\right)^{1 / 2} g^{L},
$$

while the differences $\Delta_{i} \equiv E_{i}-E_{0}$ for the higher excited states $(i>1)$ are finite for $L \rightarrow \infty$.

We consider a quench protocol in which $\lambda$ is suddenly varied. To define the general DFSS laws, we proceed as in Sec. II. First, we identify the relevant scaling variables. Arguments analogous to those reported in Ref. [25] lead us to introduce the following quantities:

$$
\kappa(\lambda)=\frac{2 m_{0} \lambda L^{b}}{\Delta(L)}, \quad \theta=t \Delta(L),
$$

where $b=1$ for the homogenous perturbation $P$ of Eq. (19), and $b=0$ for the local perturbation $P_{l}=-\sigma_{1}^{(3)}$ appearing in Eq. (26). In particular, $\kappa(\lambda)$ is the ratio between the energy associated with the longitudinal-field perturbation, which is approximately $2 m_{0} \lambda L^{b}$, and the energy difference $\Delta(L)$ of the two lowest states at $\lambda=0$. Then, we may put forward the following DFSS for the magnetization:

$$
M\left(t, L, \lambda_{0}, \lambda, L\right)=m_{0} \mathcal{M}_{f o}\left(\theta, \kappa_{0}, \kappa\right),
$$

where $\kappa_{0} \equiv \kappa\left(\lambda_{0}\right), \kappa \equiv \kappa(\lambda)$, and $m_{0}$ is given by Eq. (32). DFSS is expected to hold for any $g<1$. In particular, the scaling function $\mathcal{M}_{f o}\left(\theta, \kappa_{0}, \kappa\right)$ is expected to be independent of $g$, apart from trivial normalizations of the arguments. 
The previous scaling relations can be straightforwardly extended to any FOQT, by identifying the scaling variable $\kappa(\lambda)$ as the ratio $\lambda E_{p}(L) / \Delta(L)$, where $E_{p}(L)$ is the energy associated with the perturbation $P$ and $\Delta(L)$ is the energy difference between the two lowest states at the transition point. The second scaling variable $\theta$ is always defined as in Eq. (35).

In the case of the quantum Ising ring, some scaling functions can be exactly computed, exploiting a two-level truncation of the spectrum [25,36]. As shown in Ref. [25], in the long-time limit and for large systems, the scaling properties in a small interval around $\lambda=0$ (more precisely, for $m_{0}|\lambda| \ll \Delta_{2}$ ) are captured by a two-level truncation, which only takes into account the two nearly degenerate lowest-energy states. The effective evolution is determined by the Schrödinger equation [25]

$$
i \frac{d}{d t} \Psi(t)=H_{2}(\lambda) \Psi(t),
$$

where $\Psi(t)$ is a two-component wave function, the components of which correspond to the states $|+\rangle$ and $|-\rangle$, and

$$
H_{2}(\lambda)=-\beta \sigma^{(3)}+\delta \sigma^{(1)} .
$$

Here $\beta=m_{0} \lambda L^{b}$ and $\delta=\Delta / 2$, such that $\kappa(\lambda)=2 \beta / \Delta$ and $\theta=2 t \delta$. The initial condition is given by the ground state of $H_{2}\left(\lambda_{0}\right)$, i.e., by

$$
\Psi(t=0)=\sin \left(\alpha_{0} / 2\right)|-\rangle+\cos \left(\alpha_{0} / 2\right)|+\rangle,
$$

with $\tan \alpha_{0}=\kappa_{0}^{-1}$. The quantum evolution can be easily obtained by diagonalizing $H_{2}(\lambda)$, obtaining the eigenstates

$$
\begin{gathered}
|0\rangle=\sin (\alpha / 2)|-\rangle+\cos (\alpha / 2)|+\rangle, \\
|1\rangle=\cos (\alpha / 2)|-\rangle-\sin (\alpha / 2)|+\rangle,
\end{gathered}
$$

where $\tan \alpha=\kappa^{-1}$, and the eigenvalue difference

$$
\Delta_{\kappa} \equiv E_{1}-E_{0}=\Delta \sqrt{1+\kappa^{2}} .
$$

Then, apart from an irrelevant phase, the time-dependent state evolves as

$$
|\Psi(t)\rangle=\cos \left(\frac{\alpha_{0}-\alpha}{2}\right)|0\rangle+e^{-i \Delta_{\kappa} t} \sin \left(\frac{\alpha_{0}-\alpha}{2}\right)|1\rangle .
$$

The magnetization is obtained by computing the expectation value $\left\langle\Psi(t)\left|\sigma^{(3)}\right| \Psi(t)\right\rangle$. It gives for the dynamic scaling function defined in Eq. (36)

$$
\begin{aligned}
\mathcal{M}_{f o}\left(\theta, \kappa_{0}, \kappa\right)= & \cos \left(\alpha-\alpha_{0}\right) \cos \alpha \\
& +\cos \left(\theta \sqrt{1+\kappa^{2}}\right) \sin \left(\alpha-\alpha_{0}\right) \sin \alpha .
\end{aligned}
$$

The approach to the asymptotic result is expected to be exponential in the size of the system.

Figure 7(a) shows the behavior of the function $\mathcal{M}_{f o}\left(\theta, \kappa_{0}, \kappa\right)$, for fixed $\kappa_{0}$ and $\kappa$ and varying $\theta$, which displays the characteristic Rabi oscillations naturally emerging in the dynamics of a two-level system. Prediction (44) is also compared with the estimates of the magnetization obtained in numerical simulations. Numerical data are very close to
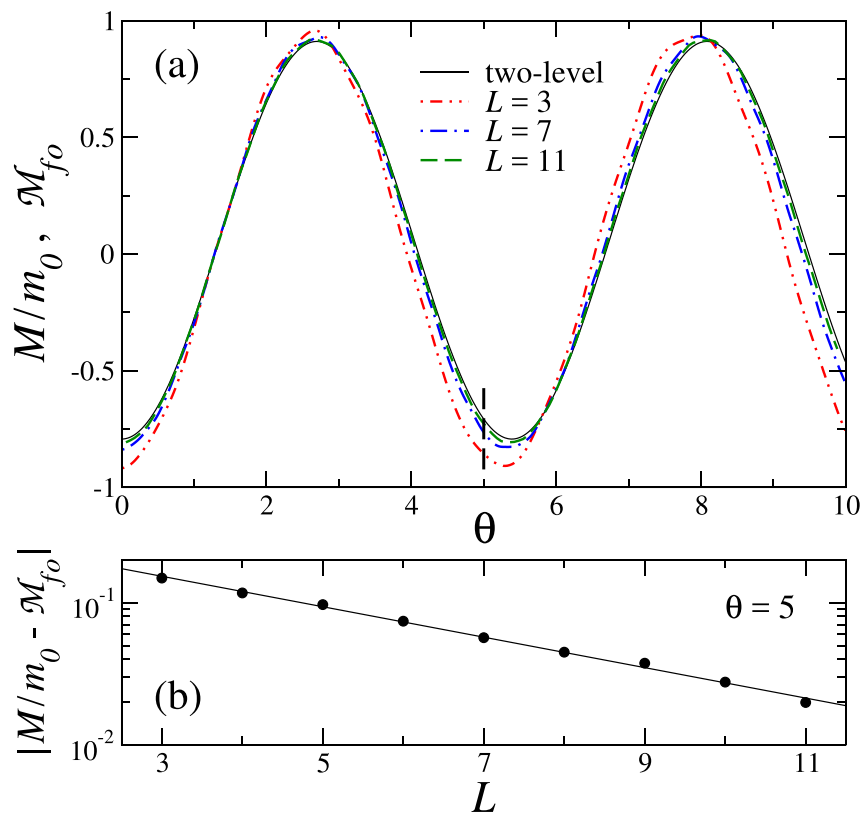

FIG. 7. (a) Plot of the ratio $M / m_{0}$ for various system sizes (color dashed curves) and of the corresponding scaling function $\mathcal{M}_{f o}\left(\theta, \kappa_{0}, \kappa\right)$, reported in Eq. (44) (black dashed curve), as functions of the rescaled time $\theta$, for fixed $\kappa_{0}=-1.3, \kappa=0.6$. Results are for the Ising ring at $g=0.9$. (b) Difference between the numerically computed $M / m_{0}$ and the function $\mathcal{M}_{f o}$, as a function of $L$ at fixed $\theta=5$. The straight line corresponds to an exponential fit of the data.

$\mathcal{M}_{\text {fo }}$ already for small chain lengths, even if data are obtained at $g=0.9$, thus relatively close to the CQT. More precisely, as shown in panel (b), scaling corrections to the two-level scaling prediction are exponentially suppressed with $L$. The nice agreement confirms that, in the DFSS limit, the dynamics can be faithfully approximated by truncating the Hilbert space to the two lowest-energy states. If $\lambda$ and $\lambda_{0}$ have opposite sign (a condition that is not necessary to observe DFSS), the two states essentially correspond to the ground states of the initial and the final Hamiltonian $H\left(\lambda_{0}\right)$ and $H(\lambda)$, respectively.

Finally we report the average work (10), computed within the two-level approximation:

$$
\mathcal{L} \equiv\langle W\rangle=-\frac{\Delta}{2} \delta_{\lambda} \frac{\kappa_{0}^{2}}{\sqrt{1+\kappa_{0}^{2}}} .
$$

See also Ref. [37] for related calculations within the two-level model.

\section{SUMMARY AND CONCLUSIONS}

We have considered the dynamics of a quantum system subject to a sudden change of a Hamiltonian parameter. Close to a quantum transition, a DFSS behavior emerges from the interplay of the parameters involved in the quench protocol and the size of the system. In particular, we have considered a generic Hamiltonian $H(\lambda)=H_{c}+\lambda P$, with $\left[H_{c}, P\right] \neq 0$, and focused on a sudden change of the parameter $\lambda$ assuming that the pre- and postquench Hamiltonians remain in the critical regime of a quantum transition. The DFSS limit is defined as the large-size and large-time limit, keeping appropriate 
scaling variables fixed. At CQTs the scaling variables are the combinations $t L^{-z}, \kappa_{0}=\lambda_{0} L^{y_{\lambda}}$, and $\kappa=\lambda L^{y_{\lambda}}$, where $z$ and $y_{\lambda}$ are appropriate critical exponents, i.e., $z$ is the dynamic exponent characterizing the size behavior of the energy gap, and $y_{\lambda}$ is the RG dimension of the parameter $\lambda$. Note that, for relevant perturbations for which $y_{\lambda}>0$, the parameters $\lambda_{0}$ and $\lambda$ have both a zero limit in the scaling regime, thereby guaranteeing that the system is always in the critical regime. It is also possible to include the effect of a small finite temperature, assuming a Gibbs ensemble as initial condition, by adding the scaling variable $\rho=T L^{z}$ as an additional argument of the DFSS functions. The general theory applies also to FOQTs with the only change that $\kappa$ should be defined as the ratio $\lambda E_{p}(L) / \Delta(L)$, where $E_{p}(L)$ is the energy associated with the perturbation $P$ and $\Delta(L)$ is the energy difference between the two lowest-energy states. In this case, it is possible for $\kappa(L)$ to depend exponentially on $L$ as a consequence of the finite-size behavior of the energy gap. We stress that the scaling arguments we have presented are quite general. Thus, they are expected to apply to generic CQTs and FOQTs in any spatial dimension.

We have verified the DFSS theory in the quantum Ising chain, the paradigmatic model undergoing FOQTs and CQTs, when varying its parameters. In particular, we have considered quench protocols associated with changes of a longitudinal magnetic field coupled to the order-parameter spin operator. We have presented analytical and numerical results for the off-equilibrium behavior of several quantities, including the magnetization, the Loschmidt echo, and the bipartite entanglement entropy. The results fully support the predictions of the DFSS theory we put forward.

A related important issue regards thermalization, that is, whether the system has a local thermal-like behavior at an asymptotically long time after the quench. Understanding under which circumstances this occurs is a highly debated issue [16], which lies outside the purpose of our analysis, being related to the integrability properties of the Hamiltonian $H_{c}$, the mutual interplay of interactions and inhomogeneities, and the nature of the spectrum. Naive scaling arguments suggest that, if the quantum evolution leads to an effective thermalization, the eventual effective temperature scales as $T \approx L^{-z} f_{T}\left(\kappa, \delta_{\lambda}\right)$. More likely, an effective thermalization may emerge in the large-volume limit (of nonintegrable systems), keeping the parameters $\lambda_{0}$ and $\lambda$ fixed, i.e., in the limit $\kappa \rightarrow \infty$, when the energy provided to the system grows as the volume, as argued at the end of Sec. II.

Finally we comment on the fact that, as foreseen by the outcomes of our numerical simulations, it is likely that the general DFSS theory following a quantum quench, described in Sec. II, can be verified even with systems of relatively small size (i.e., of the order of ten spins). Therefore, given the need for high accuracies without necessarily reaching scalability to large sizes, we believe that the available technology for probing the coherent quantum dynamics of interacting systems, such as with ultracold atoms in optical lattices [38], trapped ions [39-42], as well as Rydberg atoms in arrays of optical microtraps [43], could offer a unique playground where this theory can be reliably tested.
[1] J. von Neumann, Beweis des ergodensatzes und des H-theorems in der neuen mechanik, Z. Phys. 57, 30 (1929).

[2] W. Pauli and M. Fierz, Über das H-theorem in der quantenmechanik, Z. Phys. 106, 572 (1937).

[3] T. Niemeijer, Some exact calculations on a chain of spins $1 / 2$, Physica 36, 377 (1967).

[4] P. Mazur, Non-ergodicity of phase functions in certain systems, Physica 43, 533 (1968).

[5] E. Barouch, B. M. McCoy, and M. Dresden, Statistical mechanics of the XY model. I, Phys. Rev. A 2, 1075 (1970).

[6] E. Barouch, B. M. McCoy, and M. Dresden, Statistical mechanics of the XY model. II. spin-correlation functions, Phys. Rev. A 3, 786 (1971).

[7] I. Bloch, Quantum coherence and entanglement with ultracold atoms in optical lattices, Nature (London) 453, 1016 (2008).

[8] I. M. Georgescu, S. Ashhab, and F. Nori, Quantum simulation, Rev. Mod. Phys. 86, 153 (2014).

[9] M. Greiner, O. Mandel, T. Esslinger, T. W. Hänsch, and I. Bloch, Quantum phase transition from a superfluid to a Mott insulator in a gas of ultracold atoms, Nature (London) 415, 39 (2002).

[10] T. Kinoshita, T. Wenger, and D. S. Weiss, A quantum Newton's cradle, Nature (London) 440, 900 (2006).

[11] S. Hofferberth, I. Lesanovsky, B. Fischer, T. Schumm, and J. Schmiedmayer, Non-equilibrium coherence dynamics in onedimensional Bose gases, Nature (London) 449, 324 (2007).

[12] S. Trotzky, Y.-A. Chen, A. Flesch, I. P. McCulloch, U. Schollwöck, J. Eisert, and I. Bloch, Probing the relaxation towards equilibrium in an isolated strongly correlated onedimensional Bose gas, Nat. Phys. 8, 325 (2012).

[13] M. Cheneau, P. Barmettler, D. Poletti, M. Endres, P. Scbauß, T. Fukuhara, C. Gross, I. Bloch, C. Kollath, and S. Kuhr, Lightcone-like spreading of correlations in a quantum many-body system, Nature (London) 481, 484 (2012).

[14] M. Gring, M. Kuhnert, T. Langen, T. Kitagawa, B. Rauer, M. Schreitl, I. Mazets, D. A Smith, E. Demler, and J. Schmiedmayer, Relaxation and prethermalization in an isolated quantum system, Science 337, 1318 (2012).

[15] A. Polkovnikov, K. Sengupta, A. Silva, and M. Vengalattore, Colloquium: Nonequilibrium dynamics of closed interacting quantum systems, Rev. Mod. Phys. 83, 863 (2011).

[16] R. Nandkishore and D. A. Huse, Many body localization and thermalization in quantum statistical mechanics, Annu. Rev. Condens. Matter Phys. 6, 15 (2015).

[17] A. Chiocchetta, M. Tavora, A. Gambassi, and A. Mitra, Shorttime universal scaling and light-cone dynamics after a quench in an isolated quantum system in $d$ spatial dimensions, Phys. Rev. B 94, 134311 (2016).

[18] P. Calabrese and J. Cardy, Quantum quenches in $1+1$ dimensional conformal field theories, J. Stat. Mech. (2016) 064003.

[19] M. Heyl, Dynamical quantum phase transitions: a review, Rep. Prog. Phys. 81, 054001 (2018).

[20] M. Campostrini, A. Pelissetto, and E. Vicari, Finite-size scaling at quantum transitions, Phys. Rev. B 89, 094516 (2014). 
[21] S. Sachdev, Quantum Phase Transitions (Cambridge University, Cambridge, England, 1999).

[22] M. Campostrini and E. Vicari, Equilibrium and off-equilibrium trap-size scaling in one-dimensional ultracold bosonic gases, Phys. Rev. A 82, 063636 (2010).

[23] M. Campisi, P. Hänggi, and P. Talkner, Colloquium: Quantum fluctuation relations: Foundations and applications, Rev. Mod. Phys. 83, 771 (2011).

[24] P. Calabrese, M. Caselle, A. Celi, A. Pelissetto, and E. Vicari, Nonanalyticity of the Callan-Symanzik $\beta$-function of two-dimensional $\mathrm{O}(N)$ models, J. Phys. A 33, 8155 (2000); M. Caselle, M. Hasenbusch, A. Pelissetto, and E. Vicari, Irrelevant operators in the two-dimensional Ising model, ibid. 35, 4861 (2002).

[25] A. Pelissetto, D. Rossini, and E. Vicari, Off-equilibrium dynamics driven by localized time-dependent perturbations at quantum phase transitions, Phys. Rev. B 97, 094414 (2018).

[26] D. Rossini, S. Suzuki, G. Mussardo, and G. E. Santoro, Effective Thermal Dynamics Following a Quantum Quench in a Spin Chain, Phys. Rev. Lett. 102, 127204 (2009).

[27] P. Calabrese, F. H. L. Essler, and M. Fagotti, Quantum Quench in the Transverse Field Ising Chain, Phys. Rev. Lett. 106, 227203 (2011).

[28] M. Heyl, A. Polkovnikov, and S. Kehrein, Dynamical Quantum Phase Transitions in the Transverse-Field Ising Model, Phys. Rev. Lett. 110, 135704 (2013).

[29] G. Delfino and J. Viti, On the theory of quantum quenches in near-critical systems, J. Phys. A 50, 084004 (2017).

[30] C. Holzhey, F. Larsen, and F. Wilczek, Geometric and renormalized entropy in conformal field theory, Nucl. Phys. B 424, 443 (1994).

[31] P. Calabrese and J. Cardy, Entanglement entropy and quantum field theory, J. Stat. Mech. (2004) P06002.

[32] B.-Q. Jin and V. E. Korepin, Quantum spin chain, Toeplitz determinants and the Fisher-Hartwig conjecture, J. Stat. Phys. 116, 79 (2004).
[33] P. Calabrese, J. Cardy, and I. Peschel, Corrections to scaling for block entanglement in massive spin chains, J. Stat. Mech. (2010) P09003.

[34] P. Pfeuty, The one-dimensional Ising model with a transverse field, Ann. Phys. 57, 79 (1970).

[35] G. G. Cabrera and R. Jullien, Universality of Finite-Size Scaling: Role of the Boundary Conditions, Phys. Rev. Lett. 57, 393 (1986); Role of the boundary conditions in the finite-size Ising model, Phys. Rev. B 35, 7062 (1987).

[36] M. Campostrini, J. Nespolo, A. Pelissetto, and E. Vicari, Finitesize Scaling at First-order Quantum Transitions, Phys. Rev. Lett. 113, 070402 (2014).

[37] E. Mascarenhas, H. Bracança, R. Dorner, M. F. Santos, V. Vedral, K. Modi, and J. Goold, Work and quantum phase transitions: Quantum Latency, Phys. Rev. E 89, 062103 (2014).

[38] J. Simon, W. S. Bakr, R. Ma, M. E. Tai, P. M. Preiss, and M. Greiner, Quantum simulation of antiferromagnetic spin chains in an optical lattice, Nature (London) 472, 307 (2011).

[39] K. Kim, M.-S. Chang, S. Korenblit, R. Islam, E. E. Edwards, J. K. Freericks, G.-D. Lin, L.-M. Duan, and C. Monroe, Quantum simulation of frustrated Ising spins with trapped ions, Nature (London) 465, 590 (2010).

[40] E. E. Edwards, S. Korenblit, K. Kim, R. Islam, M.-S. Chang, J. K. Freericks, G.-D. Lin, L.-M. Duan, and C. Monroe, Quantum simulation and phase diagram of the transverse-field Ising model with three atomic spins, Phys. Rev. B 82, 060412(R) (2010).

[41] P. Richerme, Z.-X. Gong, A. Lee, C. Senko, J. Smith, M. FossFeig, S. Michalakis, A. V. Gorshkov, and C. Monroe, Non-local propagation of correlations in quantum systems with long-range interactions, Nature (London) 511, 198 (2014).

[42] P. Jurcevic, B. P. Lanyon, P. Hauke, C. Hempel, P. Zoller, R. Blatt, and C. F. Roos, Quasiparticle engineering and entanglement propagation in a quantum many-body system, Nature (London) 511, 202 (2014).

[43] H. Labuhn, D. Barredo, S. Ravets, S. de Leseleuc, T. Macri, T. Lahaye, and A. Browaeys, Tunable two-dimensional arrays of single Rydberg atoms for realizing quantum Ising models, Nature (London) 534, 667 (2016). 